\title{
Genetic depletion studies define receptor usage by virulent hantaviruses in human endothelial cells
}

M. Eugenia Dieterle ${ }^{1}$, Carles Solà-Riera ${ }^{2}$, Rohit K. Jangra ${ }^{1}$, Chunyan Ye ${ }^{3}$, Samuel M.

Goodfellow $^{3}$, Eva Mittler ${ }^{1}$, Ezgi Kasikci ${ }^{1}$, Steven B. Bradfute ${ }^{3}$, Jonas Klingström², Kartik Chandran $^{1 *}$

${ }^{1}$ Department of Microbiology and Immunology, Albert Einstein College of Medicine, Bronx, NY 10461, USA

${ }^{2}$ Center for Infectious Medicine, Department of Medicine Huddinge, Karolinska Institutet, 141 86 Stockholm, Sweden

${ }^{3}$ University of New Mexico Health Science Center, Center for Global Health, Department of Internal Medicine, Albuquerque, NM 87131, USA

*Corresponding author. kartik.chandran@einsteinmed.org

\section{Competing Interest Statement}

K.C. and R.K.J. are named co-inventors on US patent 10,105,433 covering PCDH1 as a target for anti-hantavirus treatments. K.C. is a member of the scientific advisory boards of Integrum Scientific, LLC and the Pandemic Security Initiative of Celdara Medical, LLC.

\section{Author Contributions}

M.E.D. and K.C. conceived the study. M.E.D, C.S-R., R.K.J., C.Y., S.M.G., E.M., E.K. performed experimentation. S.B.B., J.K., K.C. secured funding and provided oversight. M.E.D., R.K.J., K.C. wrote the paper with contributions from all the authors. 


\begin{abstract}
Hantaviruses are a large group of RNA viruses that include known epidemic threats and other agents poised for emergence. Several rodent-borne hantaviruses cause zoonoses accompanied by severe illness and death. However, assessments of zoonotic risk and the development of countermeasures alike are challenged by our limited knowledge of the molecular mechanisms of hantavirus infection, including the identities of cell entry receptors and their roles in influencing viral host range and virulence. Previous work has implicated several cell-surface molecules, most notably $\beta 3$ - and $\beta 1$-containing integrin heterodimers, decay-accelerating factor (DAF), and the cadherin superfamily protein protocadherin-1 (PCDH1), in hantavirus entry in endothelial cells, the major targets of viral infection in humans. Despite the fact that $\beta 3 / \beta 1$ integrins have been presumed to be the major hantavirus entry receptors for over two decades, rigorous genetic evidence supporting their requirement, and that of DAF as an entry cofactor, is lacking. Here, we used CRISPR/Cas9 engineering to knock out four candidate hantaviral receptors, singly and in combination, in a human endothelial cell line that recapitulates the properties of primary microvascular endothelial cells. PCDH1 loss substantially reduced entry and infection by a subset of hantaviruses endemic to the Americas. In contrast, the loss of $\beta 3$ integrin, $\beta 1$ integrin, and/or DAF had little or no effect on entry by any of a large panel of hantaviruses tested. We conclude that the major host molecules necessary for endothelial cell entry by PCDH1-independent hantaviruses remain to be discovered.
\end{abstract}




\section{Text}

Hantaviruses are a large group of enveloped viruses with segmented negative-strand RNA genomes whose members infect a wide range of mammalian hosts (1). The zoonotic transmission of some rodent-borne hantaviruses is associated with two major diseases in humans, hemorrhagic fever with renal syndrome (HFRS) and hantavirus cardiopulmonary syndrome (HCPS), in endemic regions of Europe/Asia and North/South America, respectively. Most HFRS cases are associated with the 'Old-World hantaviruses' Hantaan virus (HTNV), Seoul virus (SEOV), Dobrava-Belgrade virus (DOBV) and Puumala virus (PUUV), whereas HCPS is primarily caused by the 'New-World hantaviruses' Andes virus (ANDV) and Sin Nombre virus (SNV). Although humans are typically dead-end hosts for rodent-borne hantaviruses, several incidents of ANDV person-to-person transmission, including a recent superspreader event, have been documented, underscoring the epidemic threat posed by hantaviruses $(2,3)$.

Integrins $(\alpha \mathrm{V} \beta 3, \alpha 5 \beta 1, \alpha \mathrm{M} \beta 2$ and $\alpha \mathrm{X} \beta 2)$ and components of the complement system (e.g., decay accelerating factor, DAF) have been proposed as candidate hantavirus entry factors (4-8). $\beta 3$ - and $\beta 1$-containing integrins have been presumed to be the major receptors for virulent and avirulent hantaviruses, respectively, for over two decades $(5,9,10)$. More recently, we identified protocadherin-1 (PCDH1) as a critical entry determinant for New-World hantaviruses (11). However, evidentiary support for each of the above putative receptors differs considerably. $\mathrm{PCDH} 1$ was identified in a comprehensive genetic screen for ANDV entry factors. Its genetic depletion in human microvascular endothelial cells, the major targets of infection in vivo, inhibited viral entry and infection. Further, genetic ablation of PCDH1 in Syrian hamsters reduced ANDV multiplication and protected them from lethal HCPS-like disease (11). By contrast, none of the other candidate receptors were observed as hits in two independent genetic screens for hantavirus host factors (11-13). To our knowledge, their requirement has also not been rigorously evaluated via genetic approaches in physiologically relevant in vitro and in vivo models.

Here, we used a genetic depletion/complementation strategy to investigate hantavirus receptor requirements in a human microvascular endothelial cell line, TIME, a genetically 
manipulable model for primary endothelial cells (14). We first confirmed that TIME cells resembled primary human umbilical vein endothelial cells (HUVEC)-the standard model for in vitro hantavirus studies-in expressing key endothelial surface markers as well as the candidate hantavirus receptors (Fig 1a). Further, TIME cells were susceptible to hantavirus entry mediated by divergent $\mathrm{Gn} / \mathrm{Gc}$ proteins (Fig 2), and ANDV Gn/Gc-dependent entry in these cells was selectively inhibited by a PCDH1-specific monoclonal antibody, mAb-3305 (Fig 2a).

We used CRISPR/Cas9 genome engineering to knock out (KO) $P C D H 1, D A F$, and the genes encoding $\beta 3$ and $\beta 1$ integrins (ITGB3 and ITGB1) in TIME cells. Loss of specific protein expression at the cell surface and in cell extracts was verified by flow cytometry and western blotting, respectively (Fig 1b-c). We next exposed WT and KO TIME cells to recombinant vesicular stomatitis viruses carrying the Gn/Gc glycoproteins of ANDV, SNV, and HTNV (rVSV-Gn/Gc) (Fig 2b-d). An rVSV bearing the Ebola (EBOV) virus glycoprotein was used as a negative control. PCDH1 KO substantially diminished ANDV and SNV Gn/Gc-mediated infection but had no discernible effect on infection by HTNV Gn/Gc. Unexpectedly, ITGB3 and $D A F \mathrm{KOs}$ did not inhibit cell entry mediated by any of these glycoproteins (Fig $2 \mathrm{~b}-\mathrm{c})$. We extended these studies to rVSVs bearing $\mathrm{Gn} / \mathrm{Gc}$ proteins from the New-World hantaviruses Choclo virus (CHOV), Maporal virus (MPRLV), Prospect Hill virus (PHV), and the Old-World hantaviruses PUUV, SEOV, and DOBV (Fig 2d). Loss of PCDH1 substantially reduced cell entry mediated by the glycoproteins from the New-World viruses but not the Old-World viruses, in a manner that could be restored by complementation with PCDH1 cDNA. By contrast, none of these viruses displayed a requirement for ITGB3 or DAF. These findings confirm and extend the critical role played by PCDH1 in cell entry by New-World hantaviruses. However, they also contradict the claims that $\beta 3$ integrin and DAF are necessary for hantavirus entry in endothelial cells.

To account for potential receptor redundancy and cross-talk, we next evaluated double-KO populations of TIME cells (Fig 1b). PCDH1/ITGB3 and PCDH1/DAF KO cells resembled $P C D H 1 \mathrm{KO}$ cells in their susceptibility to $\mathrm{rVSV}-\mathrm{Gn} / \mathrm{Gc}$ infection (Fig 2e), indicating that the loss of PCDH1 did not unmask viral requirements for $\beta 3$ integrin or DAF, or vice versa. Further, ITGB3/DAF KO cells remained fully susceptible to viral entry (ANDV, SNV) or 
subject to only a modest ( $25 \%$ ) reduction (HTNV) (Fig 2e). Thus, the lack of viral entry phenotypes in $I T G B 3$ and $D A F$ single-KO cells cannot be explained by the functional redundancy of these two proteins.

Finally, we evaluated the single-KO TIME cells for infection with authentic hantaviruses by immunofluorescence microscopy (Fig 2f), which corroborated our findings with rVSVs. Specifically, we observed a critical role for PCDH1 in endothelial cell infection by the New-World hantaviruses ANDV, SNV, and PHV, and no apparent roles for $\beta 3$ integrin and DAF in entry by virulent New-World and Old-World hantaviruses. Concordantly, quantitative real-time PCR revealed a substantial reduction in the generation of SNV progeny genomes at 24

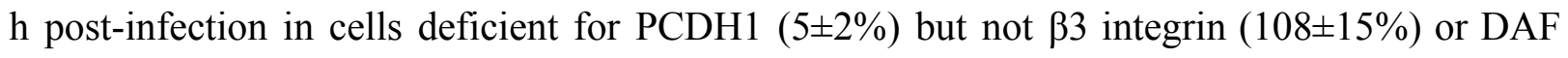
( $82 \pm 13 \%$; mean \pm SEM, $n=6$ from two independent experiments for each cell line). Moreover, and contrary to an earlier report (5), we also found no apparent role for $\beta 1$ integrin in entry by the avirulent New-World hantavirus PHV.

Our results underscore PCDH1's critical role as an entry receptor for New-World hantaviruses in endothelial cells but indicate that three other major candidate receptors ( $\beta 3$ integrin, $\beta 1$ integrin, DAF) are dispensable. We note that our results do not rule out that one or more of these proteins are involved in hantavirus entry in other cell types or that they are involved in endothelial cell subversion post-viral entry. The sum of the evidence does indicate, however, that the major host molecules necessary for entry and infection in endothelial cells by the PCDH1-independent, HFRS-causing Old-World hantaviruses remain to be discovered.

\section{Materials and methods}

Generation of KO cell populations. Gene-specific single-guide RNAs were cloned into lentiCRISPR v2 and CRISPR-Cas9 engineering was performed as described (11). Receptor-negative subpopulations of TIME cells were isolated by FACS-sorting. The targeted genomic loci in genomic DNA isolated from these subpopulations were verified by Sanger sequencing. All sequenced clones bore a frameshift inducing-indel at the targeted site. 
rVSVs and infections. rVSVs expressing a fluorescent reporter and hantavirus Gn/Gc were described previously $(11,12,15)$ or generated as described (12). Viral infectivity was measured by automated enumeration of fluorescent cells (12). 100\% relative infectivity corresponds to 18-35\% infected cells for ANDV, SNV, HTNV, EBOV, CHOV, MPRLV; 8-15\% for SEOV, DOBV, PHV and PUUV.

Hantaviruses and infections. ANDV isolate Chile-9717869, HTNV isolate 76-118, PUUV isolate Sotkamo, PHV, and SNV isolate SN77734 were used. Cells were infected with the respective virus. After $48 \mathrm{~h}$ (24 h for SNV), cells were immunostained (16). Cells were imaged and enumerated by fluorescence microscopy $(11,16)$.

Extended methods are available for this paper.

\section{Acknowledgments}

This work was supported by NIH grant R01AI132633 (to K.C.), and Swedish Research Council grant 2018-02646 (to J.K). M.E.D. was partially supported as a Latin American Fellow in the Biomedical Sciences of the Pew Charitable Trusts. S.M.G. was partially supported by the UNM HSC Infectious Disease and Inflammation Program NIH grant T32AI007538. The Einstein Flow Cytometry Core is partially supported by NIH grant P30CA013330. 


\section{Bibliography}

1. E. Mittler, et al., Hantavirus entry: Perspectives and recent advances. Adv. Virus Res. 104, 185-224 (2019).

2. V.P. Martínez, et al., "Super-Spreaders" and Person-to-Person Transmission of Andes Virus in Argentina. N. Engl. J. Med. 383, 2230-2241 (2020).

3. P.J. Padula, et al., Hantavirus pulmonary syndrome outbreak in Argentina: molecular evidence for person-to-person transmission of Andes virus. Virology 241, 323-330 (1998).

4. E. Krautkrämer, M. Zeier, Hantavirus causing hemorrhagic fever with renal syndrome enters from the apical surface and requires decay-accelerating factor (DAF/CD55). $J$. Virol. 82, 4257-4264 (2008).

5. I.N. Gavrilovskaya, M. Shepley, R. Shaw, M.H. Ginsberg, E.R. Mackow, beta3 Integrins mediate the cellular entry of hantaviruses that cause respiratory failure. Proc Natl Acad Sci USA 95, 7074-7079 (1998).

6. M.J. Raftery, et al., $\beta 2$ integrin mediates hantavirus-induced release of neutrophil extracellular traps. J. Exp. Med. 211, 1485-1497 (2014).

7. E. Popugaeva, et al., Dobrava-Belgrade hantavirus from Germany shows receptor usage and innate immunity induction consistent with the pathogenicity of the virus in humans. PLoS ONE 7, e35587 (2012).

8. T. Buranda, et al., Recognition of decay accelerating factor and alpha(v)beta(3) by inactivated hantaviruses: Toward the development of high-throughput screening flow cytometry assays. Anal. Biochem. 402, 151-160 (2010).

9. T. Raymond, E. Gorbunova, I.N. Gavrilovskaya, E.R. Mackow, P. Palese, Pathogenic Hantaviruses Bind Plexin-Semaphorin-Integrin Domains Present at the Apex of Inactive, Bent $\alpha v \beta 3$ Integrin Conformers. Proceedings of the National Academy of Sciences of the United States of America 102, 1163-1168 (2005).

10. I.N. Gavrilovskaya, T. Peresleni, E. Geimonen, E.R. Mackow, Pathogenic hantaviruses selectively inhibit beta3 integrin directed endothelial cell migration. Arch. Virol. 147, 1913-1931 (2002).

11. R.K. Jangra, et al., Protocadherin-1 is essential for cell entry by New World hantaviruses. Nature 563, 559-563 (2018).

12. L.M. Kleinfelter, et al., Haploid genetic screen reveals a profound and direct dependence on cholesterol for hantavirus membrane fusion. MBio 6, e00801 (2015).

13. J. Petersen, et al., The major cellular sterol regulatory pathway is required for Andes 
virus infection. PLoS Pathog. 10, e1003911 (2014).

14. E. Venetsanakos, et al., Induction of tubulogenesis in telomerase-immortalized human microvascular endothelial cells by glioblastoma cells. Exp. Cell Res. 273, 21-33 (2002).

15. M.M. Slough, K. Chandran, R.K. Jangra, Two point mutations in old world hantavirus glycoproteins afford the generation of highly infectious recombinant vesicular stomatitis virus vectors. MBio 10, (2019) doi:10.1128/mBio.02372-18.

16. W. Christ, J. Tynell, J. Klingström, Puumala and Andes Orthohantaviruses Cause Transient Protein Kinase R-Dependent Formation of Stress Granules. J. Virol. 94, (2020) doi:10.1128/JVI.01168-19. 
Figure 1

a
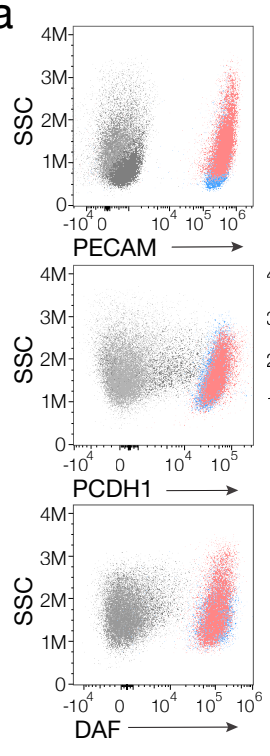

- HUVEC

C
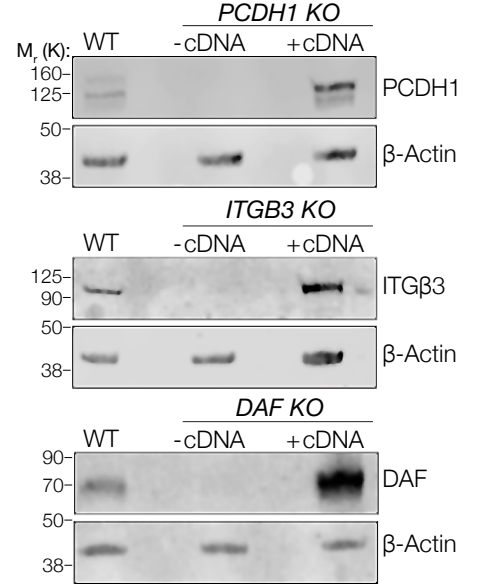

$\mathrm{b}$
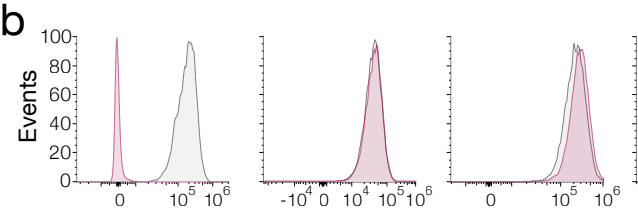

PCDH1 KO
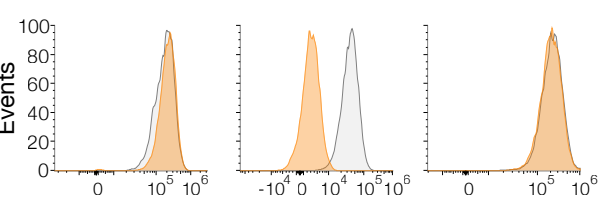

$-10^{4} \quad 0 \quad 10^{4} 10^{5}$
ITGB3 KO
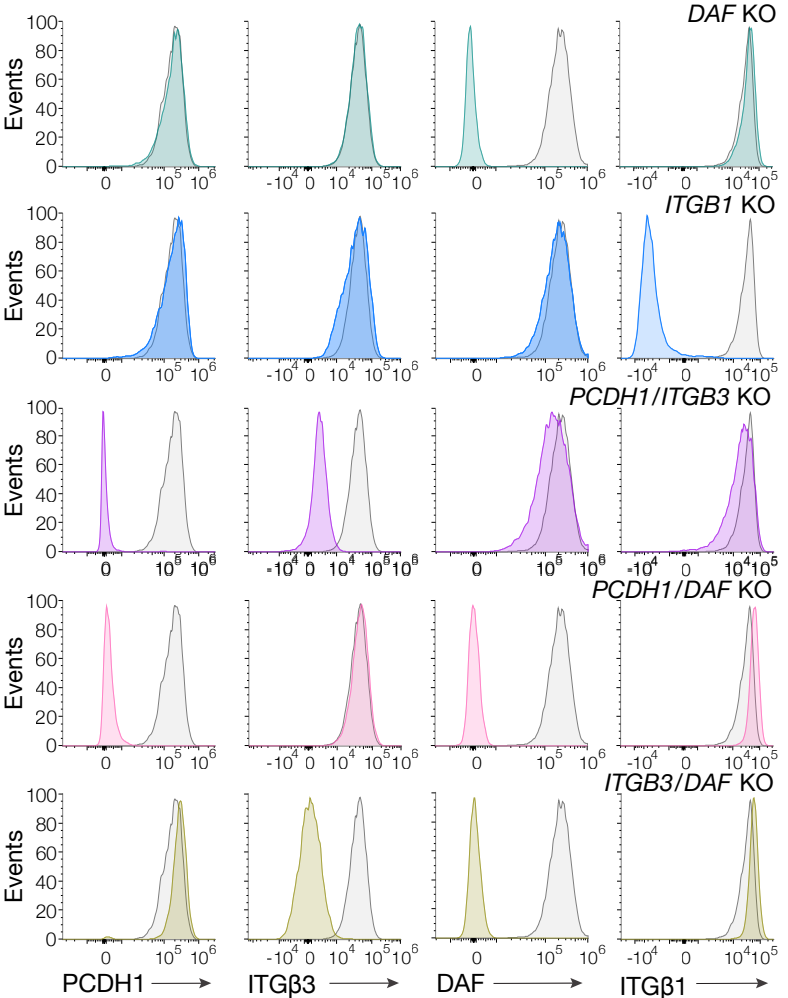
Figure 1. Suitability of TIME cells as a model to study hantavirus entry and the generation of knockout cells. (a) Upper panels, total flow cytometry plots of HUVEC and TIME cells stained for endothelial cell markers PECAM and vWF. Medium and lower panels, surface flow cytometry plots of HUVEC and TIME cells stained for the indicated markers. (b) Surface flow cytometry of WT and KO TIME cells stained as above. Histograms of WT cells are shown in gray; single- and double-KO cells are shown in color. (d) Western blot analysis of WT TIME cells and $\mathrm{KO}$ cells \pm cDNA. $\beta$-Actin was used as a loading control. 
Figure 2

a

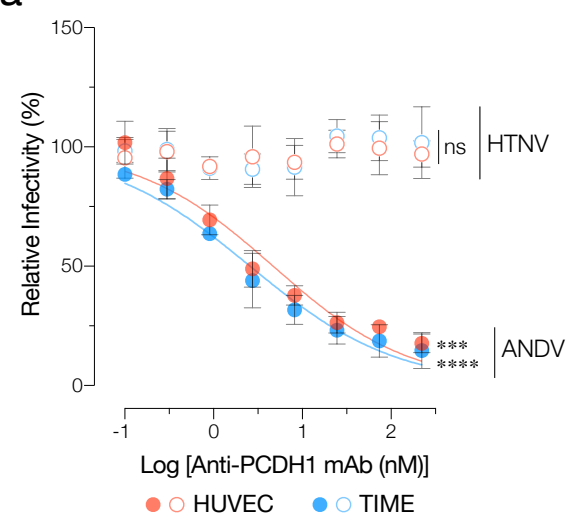

C

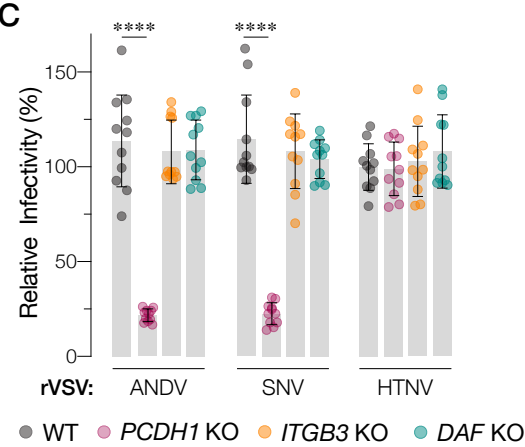

b

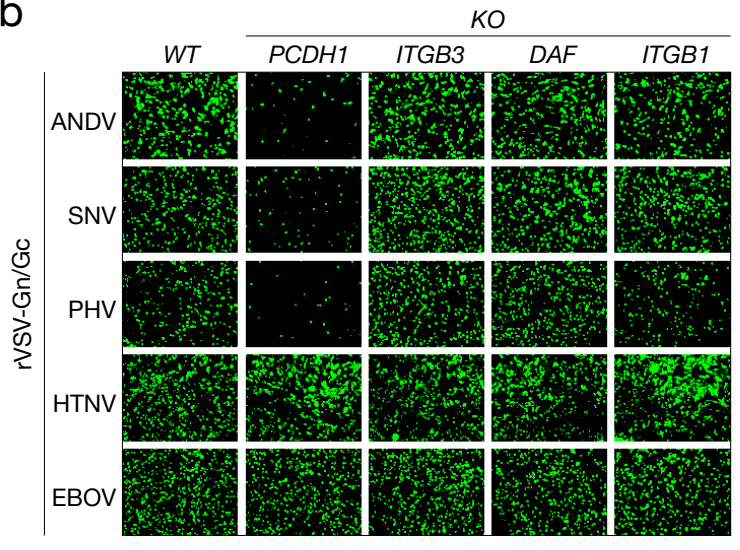

d rVSV-Gn/Gc

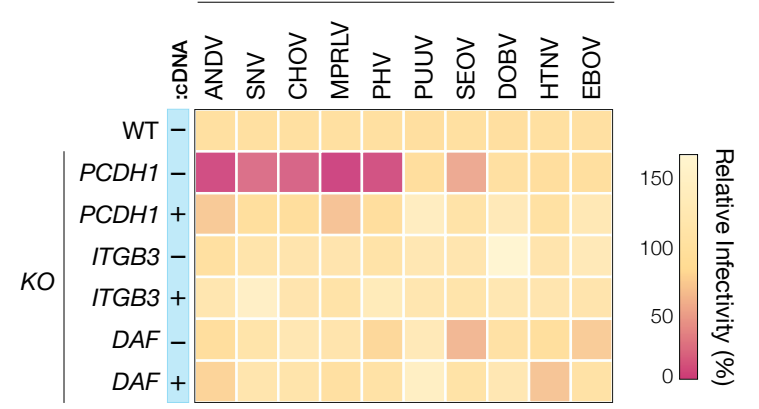

e

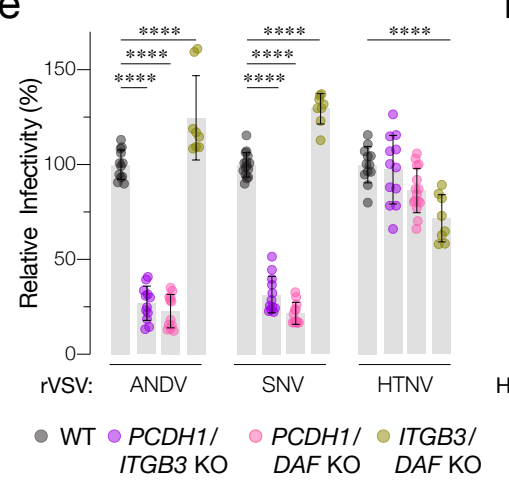

f

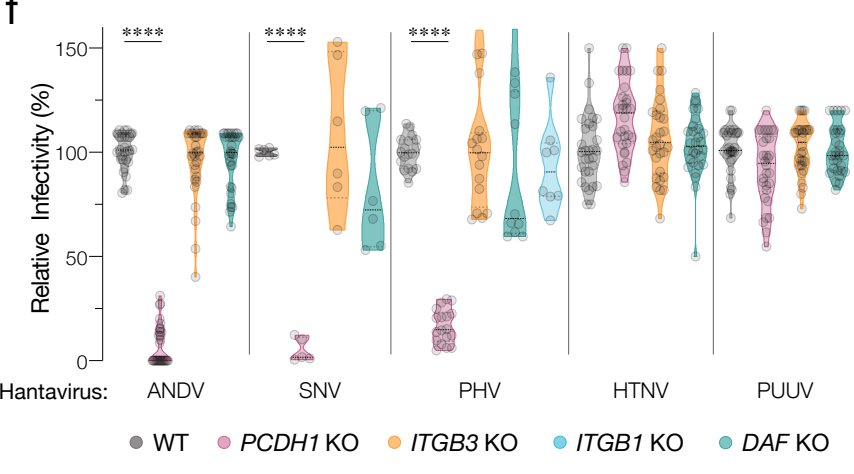


Figure 2. Hantavirus receptor requirement in endothelial cells. (a) Capacity of PCDH1 monoclonal antibody (mAb-3305) to block viral entry. HUVEC and TIME cells were preincubated with mAb-3305, and then exposed to the indicated rVSVs. TIME cells, $\mathrm{n}=6$ from 3 independent experiments, HUVEC, $\mathrm{n}=4$ from 2 independent experiments. (b) Representative images of eGFP-positive rVSV-infected WT and KO TIME cells. (c) WT and KO cells were exposed to the indicated rVSV-Gn/Gc. $n=11$ for each cell line from 3 independent experiments. (d) WT and KO cells \pm corresponding cDNA were exposed to $\mathrm{rVSV}-\mathrm{Gn} / \mathrm{Gc}$. Viral infectivities (averages from 3 independent experiments) are shown in the heatmap. (e) WT and double-KO cells were exposed to rVSV-Gn/Gc. PCDH1/ITGB3 KO, n=12; PCDH1/DAF KO, n=12; and $I T G B 3 / D A F \mathrm{KO}, \mathrm{n}=9$ from 3 independent experiments. (f) Cells were exposed to authentic hantaviruses and infected cells were manually enumerated by immunofluorescence microscopy for ANDV, HTNV, and PUUV (each point represents infectivity of the average of positive cells per field relative to WT). Data are from 2 independent experiments. PHV and SNV-infected cells were detected and enumerated by automated imaging following immunofluorescence staining. For PHV, WT and PCDH1 KO n=18, ITGB3 KO n=16 from 4 independent experiments; DAF $\mathrm{KO}, \mathrm{n}=10, I T G B 1 \mathrm{KO}$ n=8, from 3 independent experiments. For SNV WT, ITGB3 KO, DAF $\mathrm{KO}$ n=6; PCDH1 KO n=5 from 3 independent experiments. Averages \pm SD are shown. WT vs. KO comparisons by two-way ANOVA with Tukey's test (a, f) or Dunnett's test (c, e) ***, $\mathrm{P}<0.0002$; ****, $\mathrm{P}<0.0001$. Other comparisons were not significant $(\mathrm{P}>0.05, \mathrm{~ns})$. 


\section{Extended Methods}

Cells. HUVEC (C2517A-Lonza) and TIME cells (ATCC CRL4025) were cultured as described $(1,2)$.

Surface and total flow cytometry. Antibodies: AF480-Rabbit- $\alpha-v W F$ (ab195028-Abcam), PeCy7-Mouse- $\alpha-P E C A M \quad$ (563651-BD), APC-Mouse- $\alpha-\beta 1$-Integrin $\quad(559883-B D), \quad$ AF 647-Mouse- $\alpha-\beta 3$-Integrin (336407-Biolegends), PE-Mouse- $\alpha-D A F$ (555694-BD); $\alpha-P C D H 1$ mAb 3305 (2) /AF488- $\alpha$-Human (A-11013-Invitrogen). For surface staining, cells were kept at $4^{\circ} \mathrm{C}$. Foxp3/Transcription Factor Staining Buffer Kit was used for intracellular staining following the manufacturer's instructions (TNB-0607-KIT).

Western blotting. Performed as described (2). Antibodies: Mouse $\alpha-$ PCDH1 (sc-81816-Santa Cruz) 1:200; Rabbit- $\alpha-\beta 3$ Integrin (\#4702-Cell Signaling) (1:300); Mouse- $\alpha-D A F$ (NaM16-4D3-Santa Cruz) 1:200; Mouse $\alpha$ - $\beta$-Actin (sc-4778-Santa Cruz) (1:300). IRDye 680LT Goat $\alpha$-Rabbit IgG 680 or IRDye 680LT Goat $\alpha$-Mouse secondaries Abs (LI-COR, Lincoln, NE) were used at a dilution of 1:10,000, and the final blot was then imaged using a LI-COR Fc fluorescent imager.

mAb-3305 mediated inhibition of virus entry as described earlier (2). Briefly, HUVEC and TIME cells were preincubated with mAb 3305 (0-222 nM), and then exposed to rVSVs bearing ANDV or $\mathrm{HTNV} \mathrm{Gn} / \mathrm{Gc}$. $\mathrm{NH}_{4} \mathrm{Cl}(20 \mathrm{mM})$ was added 1 hour after infection. Viral infectivity was measured 14 hours post infection by automated enumeration of eGFP positive cells using a Cytation5 automated fluorescence microscope (BioTek) and analyzed using the Gen5 data analysis software (BioTek).

Generation of KO cell populations A CRISPR sgRNA was designed to target, $P C D H 1$ (2); ITGB3 5'-CCACGCGAGGTGTGAGCTCCTGC-3'; 5'-CCCCCAGATGTACCTAATGCCCA-3 and ITGB1 5'-ATACAAGCAGGGCCAAATTGTGG-3' (protospacer acceptor motif [PAM] is underlined). sgRNAs were cloned into lentiCRISPR v2 (Addgene plasmid \# 52961) and CRISPR-Cas9 engineering was performed as described (2). Receptor-negative subpopulations of TIME cells were isolated by FACS-sorting. The targeted genomic loci in genomic DNA isolated from these 
subpopulations were amplified by PCR, and the amplicons were TA-cloned into the pGEM-T vector. 15-20 clones for each KO cell population were subjected to Sanger sequencing. For each KO cell population, all sequenced clones showed an indel at the targeted site, resulting in a frameshift that brought one or more stop codons into frame.

rVSVs and infections. rVSVs expressing eGFP and bearing Gn/Gc from ANDV, SNV, HTNV, SEOV, DOBV, MPRLV, EBOV, PHV, PUUV were described previously (2-6). rVSV expressing mNeon Green fused to the VSV phosphoprotein and bearing Gn/Gc from CHOV (GenBank accession number KT983772.1) was engineered, rescued and propagated as described before (4). Viral infectivity was measured 14 hours post infection by automated enumeration of eGFP- or mNeongreen- positive cells using a Cytation5 automated fluorescence microscope (BioTek) and analyzed using the Gen5 data analysis software (BioTek). 100\% relative infectivity corresponds to $18-35 \%$ infected cells for ANDV, SNV, HTNV, EBOV, CHOV, MPRLV. 8-15\% for SEOV, DOBV, PHV, PUUV.

Hantavirus and infections. ANDV isolate Chile-9717869, HTNV isolate 76-118, PUUV isolate Sotkamo, PHV, and SNV isolate SN77734 were used. TIME cells were seeded in 24-well plates (on glass coverslips) or 96 well plate, and infected with a multiplicity of infection of 1. After 48 hours (24 hs for SNV), immunofluorescence was done as described before (7). Briefly, human polyclonal antibodies from convalescent patient serum plus anti-hantavirus nucleocapsid protein B5D9 monoclonal antibody (Progen) were used for HTNV, PUUV, ANDV. Rabbit polyclonal sera specific for SNV nucleoprotein NR-12152 (BEI Resources) was used for PHV and SNV and incubated for an hour. For HTNV, PUUV, ANDV infected cells, AF488- $\alpha-$ Human and $\alpha$-Mouse IgG antibodies (Life Technologies) were used as secondaries. For SNV and PHV, AF488- $\alpha-$ Rabbit IgG was used (Life Technologies). Counterstaining of nuclei was done with DAPI (Thermo Fisher Scientific). Cells were imaged by fluorescence microscopy (Nikon, Eclipse TE300) with a 60X objective or by automated enumeration of eGFP-positive cells using a Cytation5 automated fluorescence microscope (BioTek) as described.

SNV RNA levels. WT and KO TIME cells were harvested before infection (negative control) or at 24 hours after infection. Degenerate primers and probe were adopted from (8) based on the 
S-segment of the SNV genome. Human $\beta$-Actin primers with a VICTM/TAMRA ${ }^{\text {TM}}$-dye probe (Applied Biosystems, Cat. \# 4310881E) was used as an endogenous control. The qPCR reactions were carried out by using TaqMan ${ }^{\mathrm{TM}}$ Fast Advanced Master Mix (Applied Biosystems, Thermo Fisher Scientific) following instruction manual. Relative gene fold change was calculated by normalizing SNV to $\beta$-actin in cells using $\Delta \Delta \mathrm{Ct}$ values. Averages $\pm \mathrm{SEM}$ from two independent experiments are shown.

Statistics and reproducibility. The number of independent experiments and the measures of central tendency and dispersion used in each case are indicated in the figure legends. The testing level (alpha) was 0.05 for all statistical tests. Statistical comparisons were carried out by two-way ANOVA with a post hoc correction for family-wise error rate. All analyses were carried out in GraphPad Prism.

\section{Bibliography}

1. E. Venetsanakos, et al., Induction of tubulogenesis in telomerase-immortalized human microvascular endothelial cells by glioblastoma cells. Exp. Cell Res. 273, 21-33 (2002).

2. R.K. Jangra, et al., Protocadherin-1 is essential for cell entry by New World hantaviruses. Nature 563, 559-563 (2018).

3. M.M. Slough, K. Chandran, R.K. Jangra, Two point mutations in old world hantavirus glycoproteins afford the generation of highly infectious recombinant vesicular stomatitis virus vectors. MBio 10, (2019) doi:10.1128/mBio.02372-18.

4. L.M. Kleinfelter, et al., Haploid genetic screen reveals a profound and direct dependence on cholesterol for hantavirus membrane fusion. MBio 6, e00801 (2015).

5. A.C. Wong, R.G. Sandesara, N. Mulherkar, S.P. Whelan, K. Chandran, A forward genetic strategy reveals destabilizing mutations in the Ebolavirus glycoprotein that alter its protease dependence during cell entry. J. Virol. 84, 163-175 (2010).

6. P.F. Kerkman, et al., The generation of plasma cells and $\mathrm{CD} 27^{-} \mathrm{IgD}^{-} \mathrm{B}$ cells during Hantavirus infection are associated with distinct pathological findings. BioRxiv doi: 10.1101/723585 (2019) doi:10.1101/723585.

7. W. Christ, J. Tynell, J. Klingström, Puumala and Andes Orthohantaviruses Cause Transient Protein Kinase R-Dependent Formation of Stress Granules. J. Virol. 94, (2020) doi:10.1128/JVI.01168-19.

8. M. Kramski, et al., Detection and typing of human pathogenic hantaviruses by real-time 
bioRxiv preprint doi: https://doi.org/10.1101/2020.12.30.424861; this version posted January 4, 2021. The copyright holder for this preprint

(which was not certified by peer review) is the author/funder, who has granted bioRxiv a license to display the preprint in perpetuity. It is made available under aCC-BY-NC-ND 4.0 International license.

reverse transcription-PCR and pyrosequencing. Clin. Chem. 53, 1899-1905 (2007). 